Conclusions These preliminary and novel cost-effectiveness data indicate RFA treatment is likely to be cost-effective for patients with ongoing symptoms of APC-refractory GAVE, and could lead to substantive reductions in health care resource and also have a notable impact on a patient's state of health. As a rare disease, clinical data in this area is limited. Data from ongoing registry studies will support more sophisticated assumptions beyond expert input.

\section{PTH-040 MEDIUM TERM OUTCOMES OF ENDOSCOPIC STRETTA THERAPY IN REFRACTORY GORD- SINGLE CENTER 2- YEAR FOLLOW UP}

${ }^{1}$ YKS Viswanath, ${ }^{1}$ Nicola Maguire*, ${ }^{2}$ Anjan Dhar, ${ }^{2}$ Siby Punnoose. ${ }^{1}$ James Cook University Hospital, Middlesbrough, UK; ${ }^{2}$ Durham and Darlington NHS Foundation Trust, Darlington, UK

\subsection{6/gutjnl-2019-BSGAbstracts.65}

Background Stretta ${ }^{\circledast}$ offers a therapeutic alternative for patients suffering from refractory gastro-oesophageal reflux disease (GORD). Current evidence suggests the treatment may improve symptoms of GORD and decrease requirement for proton pump inhibitor (PPI) therapy. This is the first UK study to evaluate outcomes after Stretta therapy with 2 year follow up.

Methods Amongst 166 patients undergoing the Stretta therapy since 2014, we assessed outcomes of 50 patients where follow up was available for at least 24 months (October 2014 and February 2016) in a UK tertiary referral centre. All were assessed for suitability using endoscopy; contrast studies; and $\mathrm{pH}$ and manometry studies. Data was held in accordance with The Data Protection Act 1998. The Gastro-oesophageal Reflux Disease-Health Related Quality of Life (GERD-HRQL) was utilized to evaluate symptoms pre and post treatment. Patients were followed up by outpatient clinic appointment and telephone consultation.

Results Fifty consecutive patients were followed up for a median of 25.3 months [771 days (Range 499-1162)] following Stretta ${ }^{\circledR}$. The mean age of the cohort was 52.3 years (SD $13.9)$ and the majority were female (70\%). Seventy-two percent of patients were taking a proton pump inhibitor and $27.9 \%$ were using at least two anti-acid medications at referral. Stretta ${ }^{\circledR}$ was carried out under conscious sedation in $69.4 \%$ and general anaesthetic in 30.6\%. The mean total heartburn scores improved from 21.8 (SD 6.5) to 6.7 (SD 7.5) and regurgitation scores from 20.0 (SD 8.3) to 6.7 (SD 7.7) out of a possible 30 following Stretta ${ }^{\circledR}$. The average GERD-HRQL score improved from 46.2/75 (SD 14.2) compared to $15.2 / 75$ (SD 17.3) Dissatisfaction with GORD as measured in the GERD-HRQL decreased from 100\% to $6.2 \%$ with 3 patients showing no improvement and two late failures. No complications or readmissions occurred following the procedure.

Conclusion There are currently few effective therapeutic endoscopic procedures to anti-reflux surgery for refractory GORD. This series corroborates the value and safety of Stretta ${ }^{\circledR}$ as a viable option for selected patients instead of surgery, more so in those who are unwilling or unable to undergo surgery.

\section{PTH-041 CASE CONTROL STUDY USING A NOVEL BILIARY BRUSH FOR THE DIAGNOSIS OF DISTAL BILIARY OBSTRUCTION}

Pardeep Maheshwari, Sarah Johnson, Kofi Oppong, John Leeds, Manu Nayar. Freeman Hospital Newcastle Upon Tyne, Newcastle Upon Tyne, UK

\subsection{6/gutjnl-2019-BSGAbstracts.66}

Introduction Brush cytology is routinely performed during ERCP to assess biliary strictures but is limited by modest sensitivity (45\%). Recently a biliary brush with a new design (Infinity ${ }^{\circledR}$ brush - U.S.Endoscopy) has been introduced and preliminary reports suggest improved sensitivity. ${ }^{1}$ The brush is more abrasive and larger than conventional brushes. The aim of our study was to compare the efficacy of the new biliary brush compared with the conventional brush ( $R X$ Cytology Brush - Boston Scientific) that is routinely used in our unit.

Methods This is a matched case control study. Biliary brushings were performed with the novel brush in 50 consecutive patients from July 2017 to September 2018 for distal biliary obstruction. The cases were matched to 100 consecutive controls of the traditional brush from January 2016 to December 2016. The technique and preparation of the sample was similar for both groups. Cytology grading: C1 - inadequate, C2 = benign, $\mathrm{C} 3$ = atypia, C4 - suspicious for malignancy \& C5 = diagnostic for malignancy. Demographic data, sensitivity, specificity \& negative predictive values (NPV) were analysed for C5 alone and C4 \& C5 combined. Final diagnosis was based on a minimum follow up of 6 months. Student $t$ test \& Chi square test was used for analysis.

Results The mean age for cases was 65.4 yrs. $(\mathrm{SD}=31.4$; range $=35-91)$. The mean age for controls was 66.8 years $(\mathrm{SD}=23.5$; range $=35-88)$. M:F ratio $=$ Cases $=26: 24 \&$ controls $=48: 52$. There was no statistically significant difference between the age $(\mathrm{p}=0.86 \& 0.91) \&$ sex distribution $(p=0.79 \& 0.94)$ across the two groups.

Cases $=75 \%$ had final diagnosis of malignancy. The sensitivity, specificity and NPV for the diagnosis of malignancy using strict criteria i.e. C5 was 49\%, 100\% \& 40\% respectively. However if C4 \& C5 were combined the values were 75\%, 93\% 60\% respectively.

Controls $=75 \%$ had final diagnosis of malignancy. The sensitivity, specificity and NPV for the diagnosis of malignancy using strict criteria i.e. C5 was 52\%, 100\% \& 41\% respectively. However if C4 \& C5 were combined the values were $71 \%, 100 \% \& 56 \%$ respectively.

The mean follow up for the cases was 9.2 months and 31.7 months for the control group. There was no statistically significant difference in sensitivity $(\mathrm{p}=0.92)$, specificity $(p=0.79)$ \& NPV $(p=0.09)$ between the two brushes .

Conclusions Our data suggest that the novel brush design does not confer improved diagnostic performance in malignant biliary strictures. This highlights the difficulties of intra ductal brush sampling possibly reflecting the paucity of malignant cells within the stricture reflecting the desmoplastic nature of biliary and pancreatic malignancy.

\section{REFERENCE}

1. Shieh F, et al. World I Gastrointest Endosc 2014 Jul 16;6(7):312-7. 